\title{
One-Dimensional and Multi-Dimensional Integral Transforms of Buschman-Erdélyi Type with Legendre Functions in Kernels
}

- Authors

- $\quad$ Authors and affiliations

- Sergei M. Sitnik

- Oksana V. Skoromnik

\section{Abstract}

This paper consists of two parts. In the first part we give a brief survey of results on Buschman-Erdélyi operators, which are transmutations for the Bessel singular operator. Main properties and applications of Buschman-Erdélyi operators are outlined. In the second part of the paper we consider multi-dimensional integral transforms of Buschman-Erdélyi type with Legendre functions in kernels. Complete proofs are given in this part, main tools are based on Mellin transform properties and usage of Fox $H$-functions.

\section{Keywords}

Buschman-Erdélyi operators Multidimensional Buschman-Erdélyi operators Transmutations Mellin transform Fox $H$-function

\section{References}

1. 1.

H. Bateman, A. Erdélyi, Higher Transcendental Functions, vol. 1 (McGraw-Hill, New York, 1953)zbMATHGoogle Scholar

H. Begehr, R.P. Gilbert, Transformations, Transmutations and Kernel Functions, vols. 1, 2, Pitman Monographs and Surveys in Pure and Applied Mathematics, vol. 59 (Longman Scientific \& Technical, Harlow, 1993) Google $\underline{\text { Scholar }}$ 
R.G. Buschman, An inversion integral for a Legendre transformation. Amer. Math. Mon. 69(4), 288-289 (1962)Cross RefGoogle Scholar

5. 5.

R.G. Buschman, An inversion integral for a general Legendre transformation.

SIAM Rev. 5(3), 232-233 (1963)MathSciNetCrossRefGoogle Scholar

6. 6 .

R.W. Carroll, Transmutation and Operator Differential Equations.

Mathematics Studies, vol. 37 (North Holland, Amsterdam, 1979)Google Scholar

7. 7.

R.W. Carroll, Transmutation, Scattering Theory and Special Functions.

Mathematics Studies, vol. 69 (North Holland, Amsterdam, 1982)Google Scholar

8. 8 .

R.W. Carroll, Transmutation Theory and Applications. Mathematics Studies, vol. 117 (North Holland, Amsterdam, 1985)Google Scholar

9. 9.

R.W. Carroll, R.E. Showalter, Singular and Degenerate Cauchy

Problems (Academic, New York, 1976) Google Scholar

10. 10 .

I. Dimovski, Operational calculus for a class of differential operators. C. R. Acad. Bulg. Sci. 19(12), 1111-1114 (1966) MathSciNetGoogle Scholar

I. Dimovski, On an operational calculus for a differential operator. C. R. Acad. Bulg. Sci. 21(6), 513-516 (1968)MathSciNetzbMATHGoogle Scholar

12. 12.

I. Dimovski, Convolutional Calculus (Kluwer, Dordrecht, 1990)rossRefGoogle $\underline{\text { Scholar }}$

13. 13 .

I.H. Dimovski, V.S. Kiryakova, Transmutations, convolutions and fractional powers of Bessel-type operators via Meijer's G-function, in Complex Analysis and Applications '83. Proceedings of International Conference Varna 1983, Sofia (1985), pp. 45-66Google Scholar

A. Erdélyi, An integral equation involving Legendre functions. SIAM Rev. 12(1), 15-30 (1964)MathSciNetzbMATHGoogle Scholar 
A. Erdélyi, Some integral equations involving finite parts of divergent integrals. Glasgow Math. J. 8(1), 50-54 (1967)MathSciNetCrossRefGoogle Scholar

16. 16 .

A. Erdelyi, W. Magnus, F. Oberhettinger, F.G. Tricomi, Higher Transcendental Functions, vol. 1 (McGraw-Hill, New York, 1953)zbMATHGoogle Scholar

D.K. Fage, N.I. Nagnibida, The Problem of Equivalence of Ordinary Differential Operators (Nauka, Novosibirsk, 1977, in Russian)Google Scholar

S. Helgason, Groups and Geometric Analysis: Radon Transforms, Invariant Differential Operators and Spherical Functions (Academic, Cambridge, 1984)zbMATHGoogle Scholar

V.V. Katrakhov, S.M. Sitnik, A boundary-value problem for the steady-state Schrödinger equation with a singular potential. Soviet Math. Dokl. 3o(2), 468470 (1984)zbMATHGoogle Scholar

20. 20 .

V.V. Katrakhov, S.M. Sitnik, Factorization method in transmutation theory, in Non-classical and Mixed Type Equations (in memory of B.A. Bubnov, Ed. V.N. Vragov) (1990, in Russian), pp. 104-122Google Scholar

V.V. Katrakhov, S.M. Sitnik, Composition method for constructing $B$-elliptic, $B$ hyperbolic, and $B$-parabolic transformation operators. Russ. Acad. Sci., Dokl. Math. 50(1), 70-77 (1995) Google Scholar

V.V. Katrakhov, S.M. Sitnik, Estimates of the Jost solution to a one-dimensional Schrodinger equation with a singular potential. Dokl. Math. 51(1), 14-16 (1995)MathSciNetzbMATHGoogle Scholar

V.V. Katrakhov, S.M. Sitnik, The transmutation method and boundary-value problems for singular elliptic equations. Contemp. Math. Fund. Direct. 64(2), 211-426 (2018, in Russian)Google Scholar 
A.P. Khromov, Finite-dimensional perturbations of Volterra operators. J. Mater. Sci. 138(5), 5893-6066 (2006)MathSciNetGoogle Scholar

A.A. Kilbas, M. Saigo, H-Transforms. Theory and Applications (Chapman and Hall, Boca Raton, 2004), 400 pp.Google Scholar

A.A. Kilbas, O.V. Skoromnik, Integral transforms with the Legendre function of the first kind in the kernels on $L_{v, r}$-spaces. Integral Transform. Spec. Funct. 2o(9), 653-672 (2009)MathSciNetCrossRefGoogle Scholar

A.A. Kilbas, O.V. Skoromnik, Solution of a multidimensional integral equation of the first kind with the Legendre function in the kernel over a pyramidal domain. Dokl. Math. 80(3), 847-851 (2009)MathSciNetCrossRefGoogle Scholar

28. 28.

A.A. Kilbas, O.V. Skoromnik, Integral transforms with the legendre function of the first kind in the kernels on $\mathrm{Lv}^{-}, \mathrm{r}^{-} \mathrm{Lv}^{-}, \mathrm{r}^{-}$-spaces. Integral Transform. Spec. Funct. 20(9), 653-672 (2009)Google Scholar

A.A. Kilbas, H.M. Srivastava, J.J. Trujillo, Theory and Applications of Fractional Differential Equations (Elsevier, Amsterdam, 2006), 523 pp.zbMATHGoogle Scholar

I.A. Kipriyanov, Singular Elliptic Boundary-Value Problems (NaukaPhysmatlit, Moskow, 1997, in Russian)Google Scholar

V. Kiryakova, Generalized Fractional Calculus and Applications. Pitman Research Notes in Mathematics, vol. 301 (Longman Scientific \& Technical, Harlow, Co-publisher Wiley, New York 1994)Google Scholar

V.V. Kravchenko, Pseudoanalytic Function Theory (Birkhäuser Verlag, Basel, 2009) CrossRefGoogle Scholar 
B.M. Levitan, Generalized Translation Operators and Some of Their Applications (Israel Program for Scientific Translations, Jerusalem, 1964)Google Scholar

B.M. Levitan, Generalized Translation Operators Theory (Nauka, Moscow, 1973, in Russian)Google Scholar

B.M. Levitan, Inverse Sturm-Liouville Problems (VNU Science Press, Utrecht, 1987)CrossRefGoogle Scholar

J.L. Lions, Equations differentielles operationnelles et problémes aux limites (Springer, Berlin, 1961) CrossRefGoogle Scholar

V.A. Marchenko, Spectral Theory of Sturm-Liouville Operators (Naukova Dumka, Kiev, 1972, in Russian)Google Scholar

39. 39.

V.A. Marchenko, Sturm-Liouville Operators and Applications (AMS Chelsea Publishing, Rhode Island, 1986)rossRefGoogle Scholar

40. 40 .

O.I. Marichev, Method for Computing Integrals of Special Functions (Nauka i Technika, Minsk, 1978, in Russian)Google Scholar

41. 41.

A.M. Mathai, R.K Saxena, The H-Function with Applications in Statistics and Other Disciplines (Halsted Press, Wiley, New York, 1978)Google Scholar

42. 42.

A.P. Prudnikov, Yu. A. Brychkov, O.I. Marichev. Integrals and Series. More Special Functions, vol. 3 (Gordon and Breach, New York, 1990)Google Scholar

S.G. Samko, A.A. Kilbas, Fractional Integrals and Derivatives. Theory and Applications (Gordon and Breach, Yverdon, 1993), 1112 pp.Google Scholar

S.G. Samko, A.A. Kilbas, O.I. Marichev, Fractional Integrals and Derivatives: Theory and Applications (Gordon and Breach Science Publishers, London, 1993)zbMATHGoogle Scholar 
C. Shadan, P. Sabatier, Inverse Problems in Quantum Scattering

Theory (Springer, Berlin, 1989)Google Scholar

46. 46 .

S.M. Sitnik, Unitary and Bounded Buschman-Erdélyi Operators. Preprint, Institute of Automation and Process Control of the Soviet Academy of Sciences (1990, in Russian), Vladivostok, 44 pp.Google Scholar

S.M. Sitnik, Factorization and estimates of the norms of Buschman-Erdelyi operators in weighted Lebesgue spaces. Soviet Math. Dokladi 44(2), 641-646 (1992)zbMATHGoogle Scholar

S.M. Sitnik, Transmutations and applications, in Contemporary Studies in Mathematical Analysis, Vladikavkaz, ed. by Yu. F. Korobeinik, A.G. Kusraev (2008, in Russian), pp. 226-293Google Scholar

49. 49.

S.M. Sitnik, Factorization method for transmutations in the theory of differential equations. Vestnik Samarskogo Gosuniversiteta 67(8/1), 237-248 (2008, in Russian)Google Scholar

50. 50 .

S.M. Sitnik, Transmutations and applications: a survey (2010), arXiv:1012.3741v1, 141 pp.Google Scholar

S.M. Sitnik, A solution to the problem of unitary generalization of SoninePoisson transmutations. Belgorod State Univ. Sci. Bull. Math. Phys. 18, 5 (76), 135-153 (2010, in Russian)Google Scholar

S.M. Sitnik, Boundedness of Buschman-Erdelyi transmutations, in The Fifth International Conference "Analytical Methods of Analysis and Differential Equations" (AMADE). Mathematical Analysis, Belorussian National Academy of Sciences, Institute of Mathematics, Minsk, vol. 1 (2010, in Russian), pp. 120125 Google Scholar

53. 53.

S.M. Sitnik, Buschman-Erdélyi transmutations, classification and applications (2013). arXiv:1304.2114, 67 pp.Google Scholar 
S.M. Sitnik, Buschman-Erdélyi transmutations, classification and applications, in Analytic Methods of Analysis and Differential Equations (AMADE 2012), ed. by M.V. Dubatovskaya, S.V. Rogosin (Cambridge Scientific Publishers, Cambridge, 2013), pp. 171-201Google Scholar

55. 55.

S.M. Sitnik, Buschman-Erdélyi transmutations, their classification, main properties and applications. Sci. Bull. Belgorod State Univ. 39, 60-76 (2015) Google Scholar

56. 56.

S.M. Sitnik, A survey of Buschman-Erdélyi transmutations. Chelyabinsk Phys. Math. J. 1(4), 63-93 (2016, in Russian) Google Scholar

S.M. Sitnik, E.L. Shishkina, Method of Transmutations for Differential Equations with Bessel Operators (Moscow, Fizmatlit, 2019, in Russian), 224 pp.Google Scholar

58. 58.

H.M. Srivastava, K.C. Gupta, S.L. Goyal, The H-function of One and Two Variables with Applications (South Asian Publishers, New Delhi, 1982)zbMATHGoogle Scholar

59. 59.

Kh. Triméche, Transmutation Operators and Mean-Periodic Functions Associated with Differential Operators. Mathematical Reports, vol. 4, Part 1 (Harwood Academic Publishers, Reading, 1988)Google Scholar

I.N. Vekua, Generalized Analytic Functions (Pergamon Press, Oxford 1962)zbMATHGoogle Scholar

N. Virchenko, I. Fedotova, Generalized Associated Legendre Functions and Their Applications (World Scientific, Singapore, 2001)Cross RefGoogle Scholar 


\section{Copyright information}

(C) Springer Nature Switzerland AG 2020

\section{About this chapter}

\section{Cite this chapter as:}

Sitnik S.M., Skoromnik O.V. (2020) One-Dimensional and Multi-Dimensional Integral Transforms of Buschman-Erdélyi Type with Legendre Functions in Kernels. In: Kravchenko V., Sitnik S. (eds) Transmutation Operators and Applications. Trends in Mathematics. Birkhäuser, Cham. https://doi.org/10.1007/978-3-03035914-0_13

- First Online12 April 2020

- DOI https://doi.org/10.1007/978-3-030-35914-0 13

- Publisher Name Birkhäuser, Cham

- Print ISBN 978-3-030-35913-3

- Online ISBN 978-3-030-35914-0

- eBook PackagesMathematics and StatisticsMathematics and Statistics (R0)

- Reprints and Permissions 